\title{
Atrial fibrillation as initial presentation of myelodysplastic syndrome (Case report)
}

\author{
Talib S.H*, Kharche Jyoti **, *Sainani Rahul ***, Deshpande Shreyas***, \\ Paike Nilrohit*** \\ *Professor \& Head, **Associate Professor, ***Chief Resident \\ Department Of Medicine, Mahatma Gandhi Missions Medical College \& Hospital , Aurangabad.431003
}

\begin{abstract}
We present a case of a patient with myelodysplastic syndrome where in impreciation persists for case analysis. The cytogenetic study revealed Philadelphia negative with multiple breakpointS and isolated monosomy of chromosome 5 revealing the likely consideration for myelodysplastic myeloproliferative syndrome terminating to acute myelogenous leukaemia (unclassified RAEB category).There is absolute paucity of presentation in the world literature of cases of myelodysplastic syndrome having atrial fibrillation as the initial presentation.
\end{abstract}

\section{Introduction :}

Cardiac involvement with leukaemic blast is a well known finding in patients with acute leukaemia ${ }^{\mathbf{1 , 2}}$. However involvement of heart with conduction defects in myelodysplasia is reported rarely in the literature $\mathbf{3}^{\mathbf{3 , 4}}$ .The symptoms may be ascribed to iron overload \& side effects to therapy which are not the reasons in the present case as our patients initial presentation to hospital was with arrhythmias ( Atrial Fibrillation ).

\section{History :}

Mr KMR , 65 YRS old male was hospitalized with giddiness, palpitations, chest discomfort, weakness, dry cough, shortness of breath, easy fatiguability and lethargy which he was experiencing past 1 month prior to the hospitalization. On examination he was afebrile, Heart rate ; 150/min, irregularly irregular, ECG done suggestive of Atrial Fibrillation, Blood Pressure ; 120/80 mm Hg , Respiratory Rate ; 24/min. There was no cyanosis/jaundice. On routine blood count ; Hb-8.5gm\% ,TLC-39400/cumm, platelet-1,44,000/cumm. Peripheral smear revealed 5\% myelocyte, 5\% metamyelocyte, 15\% band cells,30\% neutrophils, 25\% lymphocyte , $2 \%$ monocyte , 3\% eosinophils , $0 \%$ blast cells. A diagnosis of chronic myeloproliferative disorder was considered. Bone marrow biopsy was undertaken along with karyotyping on the $2^{\text {nd }}$ day of hospitalization.His symptoms were attributed to anaemia \& rhythm disorder . He was provided with 1 unit of packed red cells \& Cardarone \& Digoxin for his rhythm problem . 5days later he developed mild febrile illness and breathlessness. The $\mathrm{CBC}$ at this time demonstrated $\mathrm{Hb}-9.5 \mathrm{gm} \%$,TLC-76,800/cumm, platelet1,19,000/cumm. Peripheral smear revealed 15\% myelocyte, 20\% metamyelocyte, 20\% band cells,35\% neutrophils, $10 \%$ lymphocyte , $0 \%$ monocyte , $0 \%$ eosinophils , $0 \%$ blast cells . On $7^{\text {th }}$ day of hospitalization , counts further increased to $99,790 / \mathrm{cumm}, \mathrm{Hb}-9.9 \mathrm{gm} \%$, platelets reduced to 41,000/cumm. Peripheral smear revealed 12\% myelocyte, $16 \%$ metamyelocyte, 29\% Promyelocyte, $17 \%$ band cells, $15 \%$ neutrophils, $0 \%$ lymphocyte , $0 \%$ monocyte , $2 \%$ eosinophils , $12 \%$ blast cells . The clinico-hematological diagnosis was consistent with myelodysplastic proliferative disorder terminating to myeloid leukaemia. Bone marrow biopsy was suggestive of MDS \& the karyotype revealed Monosomy of chromosome 5 and 12 along with chromosomal breakpoints at 7q11.2,8q22,21q22. Philadelphia was negative. Echocardiographic study identified $55 \%$ ejection fraction with mild pericardial effusion and heart rate irregularity. Treatment was instituted with Hydroxyurea \& supportive therapy. His condition grew worse on day 10 and developed further cardiac blocks \& hypotension with systolic blood pressure of $60 \mathrm{~mm}$ of $\mathrm{Hg}$ for which pacemaker was inserted \& vasopressor therapy was instituted. His vitals remained stable for couple of hours before developing cardiac arrest from which he couldn't be revived. PostMortem study was not available.

\section{Discussion}

Myelodysplastic syndrome are all disorders of stem cells in the bone marrow where in haematopoeisis is ineffective of the myeloid class of blood cells . In majority of cases, patient develops cytopenias because of progressive bone marrow failure and $1 / 3^{\text {rd }}$ cases with Myelodysplastic syndrome get transformed to acute myelogenous leukaemia, some have fatal outcome in few months to years .

World health organization has evolved new classification scheme (2008) based on genetic findings ${ }^{5}$ However haematocytological screening tests are still the effective tools for deciding the nature of pathology. 
Cytogenetic analysis help to evaluate impact on disease outcome of Myelodysplastic syndrome .The variables included age,gender,besides cytogenetic analysis. Cytogenetically, poor outcomes were complex \& are described as less than or equal to three abnormalities, \&/or presence or chromosome 7 anomalies . Good outcomes noted with deletion $5 \mathrm{q}$ alone, $\mathrm{Y}$ alone, deletion $20 \mathrm{q}$ alone.The present case had revealed $7 \mathrm{a} 11.2$ ,8q22,2/q22 including chromosome 7 anomaly, thus suggesting the poor outcome as also noted in our case. The patient succumbed to death within a short period of 2 weeks . The variables described by their statistical power separated such patients into distinctive subgroup of risk. High risk (8\%) describes for acute myeloid leukaemia developing in 0.2 years reported by international MDS risk analysis workshop) ${ }^{6}$

The dysplastic syndrome under new WHO system is dealt as under. ${ }^{5}$

1)RARS-Refractory anaemia with ring sideroblast with refractory

cytopenia,neutropenia,anaemia,thrombocytopenia

2)RABS-Refractory anaemia with ring sideroblast with thrombocytosis which is essence of myelodysplastic myeloproliferative disorder.

3)RAEB-Refractory anaemia with excess blast.

RAEB-1-5 to 9\% blast

RAEB-2-10 to $19 \%$ blast

Auer bodies may be seen in RAEB 2 which has a poor prognosis.

4)The category of RAEB-T. Refractory anaemia with excess blast. In transformation is eliminated and considered to have acute leukaemia with deletion of long arm of chromosome 5.

5)Myelodysplastic myeloproliferative overlap syndrome

A) $5 \mathrm{q}$ syndrome(typically seen in older women with normal or high platelet count)

B ) Refractory cytopenias of childhood.

C )Myelodysplastic unclassifiable : Where in patients with RA or RAEB occasionally present with leucocytosis or thrombocytosis instead of usual thrombocytopenia as noted in the cases.

Cardiac involvement in patients with myelodysplasia is often attributed to the presence anaemia, iron load and chemotherapy ${ }^{7,8}$. The case under discussion had presented with arrhythmia and anaemia of short duration where no chemotherapeutic agent \& or over iron dose were received by the patient. Such myelodysplastic patient usually present with symptoms attributed to cytopenias \& the clinical course progressing to bone marrow failures or transformation to leukaemias. We believe that the in conduction deformities in the patient developed due to infiltration of malignant haematopoeitic precursors with myelodysplasia . Similar type of case was reported by Mateen et al as the first case of affection of heart with myelodysplastic syndrome .Leukamic/myelodysplastic cardiac involvement in life is usually not suspected because of subclinical nature of the symptoms \& signs with this pathology ${ }^{9}$. However cardiac involvement in leukaemia extramedullary (heart)spread is relatively common ranging 37 to $44 \%^{2}$

In our reported case, extramedullary cardiac involvement by dysplastic haematopoetic precursors by itself is considered most reasonable hypothesis as the patient presented initially with atrial fibrillation . Long before the patient heralded transformation to leukaemia, yet another postulation can be entertained as explained by Bin cohette et al in $1998 .{ }^{10}$ The granulocyte macrophage colony stimulating factor overproduction may leads to autonomous colony formation in the bone marrow of patients with myelodysplastic syndrome which may also partially explain the proliferation of malignant haematopoietic cells elevation in the heart . This extramedullary cardiac spread could have heralded the conducting system leading to the devastating cardiac irregularities in the present case.

\section{References :}

[1]. Roberts WC , Bodey GP , Wertlake PT. The heart in acute leukaemia. A study of 420 autopsy cases. Am J Cardiol. 1968;21(3):388412.

[2]. Jost E, Lorenzen J, Haage P, Bos G, Beelen D, Galm O et al. Heart and muscle involvement by extra-medullary myeloid leukaemia : a case report and review of the literature. Leuk Lymphoma. 2005;46(12):1819-24.

[3]. Hatake K, Saito K, Saga T, Akashi N, Doishita K. A case of acute myelogenous leukaemia with advanced atrioventricular block and pericardial effusion caused by leukaemic cell infiltration. Jpn J Med 1982;21(2):115-19

[4]. Ottaviani G, Matturri L, Rossi L, Jones D. Sudden death due to lymphomatous infiltration of the cardiac conduction system. Cardiovasc Pathol 2003;12(2):77-81.

[5]. Wikipedia.Myelodysplastic Syndrome

[6]. Greenberg P, Cox C, LeBeau MM, Fenaux P, Morel P, Sanz G et al. International scoring system for evaluating prognosis in myelodysplastic syndromes. Blood. 1997;89(6):2079-88.

[7]. Oliva EN, Dimitrov BD, Benedetto F, D Angelo A, Nobile F. Haemoglobin level threshold for cardiac remodeling and quality of life in myelodysplastic syndrome. Leuk Res. 2005;29(10):1217-19.

[8]. Greenberg PL. Myelodysplastic syndromes:iron overload consequences and current chelating therapies. J Natl Compr Canc Netw. 2006; 4(1):91-96

[9]. Athens JWJ. Complications of haematopoietic neoplasms. Wintrobes Clinical Haematology. Vol 2.Edited by Lee GR. Philadelphia , Lea and Febiger ; 1993 : $1792-1842$.

[10]. Bincoletto C, Saad ST, Soares da Silva E, Queiroz ML. Autonomous proliferation and bcl-2 expression involving haematopoeitic cells in patients with myelodysplastic syndrome. Br J Cancer. 1998;78(5):621-24. 
Figures :

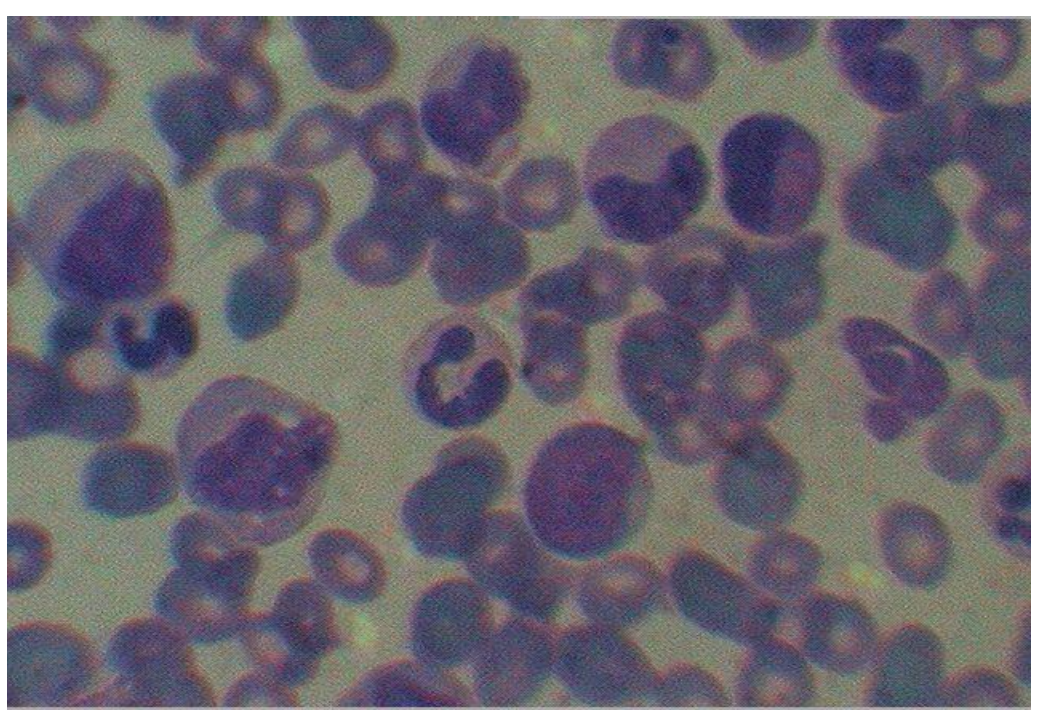

Fig: 1 Peripheral smear ( H \& E stain ) x100 showing immature cells - Myeloblast , Promyelocyte , Metamyelocyte $\&$ band forms consistent with the myeloblastic disorder.

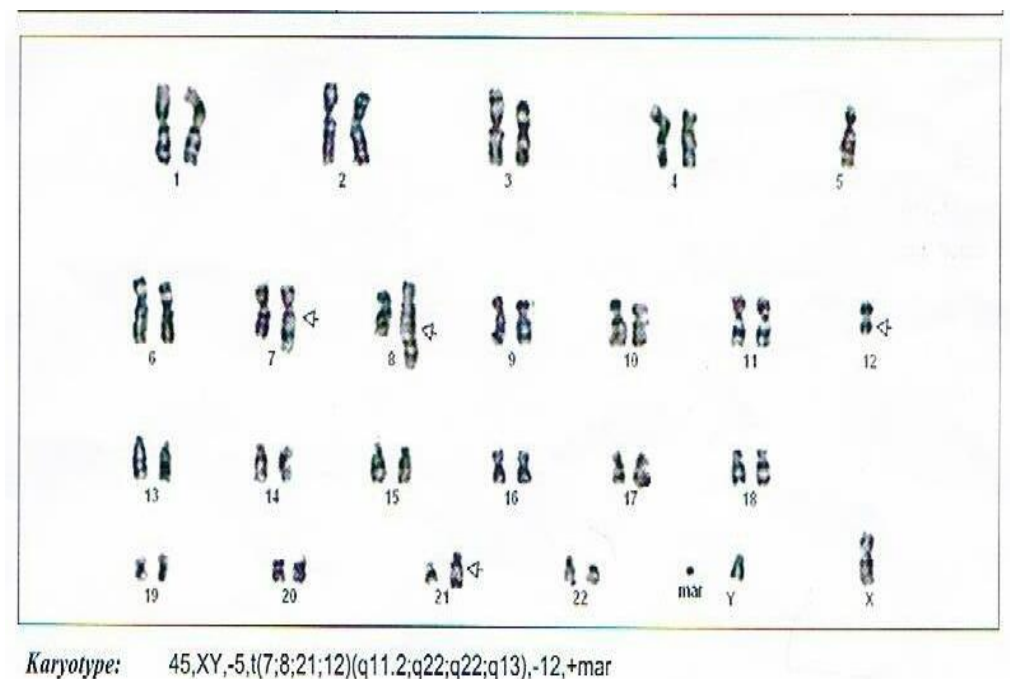

Fig 2 : Karyotyping analysis showing 45,XY,-5,t(7;8;21;12)(q11.2;q22;q22;q13),-12,-mar.

Analysis revealing a total of 42 autosomes , 2 sex chromosomes \& a marker chromosome of unknown origin . There is monosomy of chromosome $5 \& 12$ with presence of 4 break rearrangements between $7 \mathrm{q} 11.2,8 \mathrm{q} 22$, $21 \mathrm{q} 22$ \& 12q13 region . 